\title{
SOME IDEALS OF TERNARY SEMIGROUPS
}

\section{BY}

\author{
S. KAR and B.K. MAITY
}

\begin{abstract}
In this paper we characterize different types of ideals of ternary semigroup and study some interesting properties of these ideals of ternary semigroup.

Mathematics Subject Classification 2000: 20M17.

Key words: ternary semigroup, ternary group, regular ternary semigroup, prime ideal, semiprime ideal, strongly irreducible ideal, quasi-ideal, bi-ideal, weak bi-ideal.
\end{abstract}

\section{Introduction}

There is a large literature dealing with ternary operations. The notion of ternary semigroup is a natural generalization of ternary group. The notion of ideal play very important role to study the algebraic structures. In [5], Sioson studied ideal theory in ternary semigroups. He introduced the notion of prime ideal, semiprime ideal, quasi-ideal and study regular ternary semigroup by using these ideals. In [1], Dixit and Dewan studied the notions of quasi-ideal and bi-ideal in ternary semigroups. In [4], SANTIAGO developed the theory of ternary semigroups and semiheaps. In $[2,3]$, we study regular ternary semigroups, intra-regular ternary semigroups and congruences on ternary semigroup.

In this paper we study some interesting properties of various ideals of ternary semigroups.

\section{Ideals of ternary semigroups}

Definition 2.1. A non-empty set $S$ together with a ternary operation, called ternary multiplication, denoted by juxtaposition, is said to be a ternary semigroup if $(a b c) d e=a(b c d) e=a b(c d e)$, for all $a, b, c, d, e \in S$. 
Definition 2.2. A ternary semigroup $S$ is said to be commutative if $x_{1} x_{2} x_{3}=x_{\sigma(1)} x_{\sigma(2)} x_{\sigma(3)}$ for every permutation $\sigma$ of $\{1,2,3\}$ and $x_{1}, x_{2}, x_{3} \in S$.

Definition 2.3. A non-empty subset $T$ of a ternary semigroup $S$ is called a ternary subsemigroup if $t_{1} t_{2} t_{3} \in T$, for all $t_{1}, t_{2}, t_{3} \in T$.

Definition 2.4. A non-empty subset $I$ of a ternary semigroup $S$ is called

(i) a left ideal of $S$ if $S S I \subseteq I$;

(ii) a lateral ideal of $S$ if $S I S \subseteq I$;

(iii) a right ideal of $S$ if $I S S \subseteq I$;

(iv) an ideal of $S$ if $I$ is a left, a right, a lateral ideal of $S$.

An ideal $I$ of a ternary semigroup $S$ is called a proper ideal if $I \neq S$.

In general, a lateral ideal of a ternary semigroup $S$ is not an ideal of $S$. But in particular, we have the following result:

Proposition 2.5. A minimal lateral ideal of a ternary semigroup $S$ is a minimal ideal of $S$.

Proof. Let $M$ be a minimal lateral ideal of $S$. We shall show that $M$ is a minimal ideal of $S$. Let $m \in M$. Then $S m S \cup S S m S S$ is a lateral ideal of $S$ and $S m S \cup S S m S S \subseteq S M S \cup S S M S S \subseteq M$. Since $M$ is minimal, we have $M=S m S \cup S S m S S$. Now $M S S=(S m S \cup S S m S S) S S=(S m S) S S \cup$ $(S S m S S) S S \subseteq S m S \cup S S m S S \subseteq M$ and $S S M=S S(S m S \cup S S m S S)=$ $S S(S m S) \cup S S(S S m S S) \subseteq S m S \cup S S m S S \subseteq M$. This implies that $M$ is a right ideal and also a left ideal of $S$. Also $M$ is a lateral ideal of $S$. Thus $M$ is an ideal of $S$. Now it remains to show that $M$ is a minimal ideal of $S$. If possible, let $M^{\prime}$ be an ideal of $S$ such that $M^{\prime} \subseteq M$. Since $M^{\prime}$ is an ideal of $S$, it is a lateral ideal of $S$. By hypothesis, we have $M^{\prime}=M$. Consequently, $M$ is a minimal ideal of $S$.

Proposition 2.6. Let $S$ be a ternary semigroup and $a \in S$. Then the principal

(i) left ideal generated by ' $a$ ' is given by $<a>_{l}=S S a \cup\{a\}$;

(ii) right ideal generated by ' $a$ ' is given by $<a>_{r}=a S S \cup\{a\}$; 
(iii) lateral ideal generated by ' $a$ ' is given by $<a>_{m}=S a S \cup S S a S S \cup\{a\}$;

(iv) ideal generated by ' $a$ ' is given by $<a>=S S a \cup a S S \cup S a S \cup S S a S S \cup$ $\{a\}$.

Definition 2.7. A proper ideal $P$ of a ternary semigroup $S$ is called a prime ideal of $S$ if for any three ideals $A, B, C$ of $S ; A B C \subseteq P$ implies $A \subseteq P$ or $B \subseteq P$ or $C \subseteq P$.

The following theorem gives a useful characterization of a prime ideal in a ternary semigroup.

Theorem 2.8. In a ternary semigroup $S$, the following conditions are equivalent:

(i) $P$ is a prime ideal of $S$;

(ii) For $a, b, c \in S$; $a S b S c \subseteq P, a S S b S S c \subseteq P, a S S b S c S \subseteq P$ and $S a S b S S c \subseteq P$ implies $a \in P$ or $b \in P$ or $c \in P$;

(iii) For $a, b, c \in S$; $\langle a\rangle\langle b\rangle<c\rangle \subseteq P$ implies $a \in P$ or $b \in P$ or $c \in P$.

Theorem 2.9. An ideal $P$ of a commutative ternary semigroup $S$ is prime if and only if $a b c \in P$ implies that $a \in P$ or $b \in P$ or $c \in P$, for all elements $a, b, c$ of $S$.

Proof. Let $S$ be a commutative ternary semigroup.

Suppose $P$ is a prime ideal of $S$ and $a b c \in P$ for some $a, b, c \in S$. Then $(a b c) S S \subseteq P S S \subseteq P$. This implies that $a S b S c \subseteq P$, since $S$ is commutative. Similarly, $(a b c) S S S S \subseteq P S S \subseteq P$ and hence by commutativity of $S$, we have $a S S b S S c \subseteq P, a S S b S c S \subseteq P, S a S b S S c \subseteq P$. Since $P$ is a prime ideal of $S$, by Theorem 2.8, we get $a \in P$ or $b \in P$ or $c \in P$.

Conversely, let $a b c \in P$ implies that $a \in P$ or $b \in P$ or $c \in P$, for all $a, b, c$ of $S$. We have to show that $P$ is a prime ideal of $S$. Suppose $A B C \subseteq P$ for any three ideals $A, B, C$ of $S$ and $B \not \subset P, C \not \subset P$. Then there exist $b \in B$ such that $b \notin P$ and $c \in C$ such that $c \notin P$. Now for each $a \in A$, $a b c \in A B C \subseteq P$ implies that $a \in P$ and hence $A \subseteq P$. Consequently, $P$ is a prime ideal of $S$.

Example 2.10. In the commutative ternary semigroup $\mathbb{Z}^{-}$of all negative integers, the ideal $P=\left\{3 k: k \in \mathbb{Z}^{-}\right\}$is a prime ideal. 
For $\quad x y z \in P\left(x, y, z \in \mathbb{Z}^{-}\right)$

$\Longleftrightarrow \quad x y z$ is divisible by 3

$\Longleftrightarrow \quad x$ is divisible by 3 or $y$ is divisible by 3 or $z$ is divisible by 3

$\Longleftrightarrow \quad x=3 k_{1}$ or $y=3 k_{2}$ or $z=3 k_{3}$ for $k_{1}, k_{2}, k_{3} \in \mathbb{Z}^{-}$

$\Longleftrightarrow \quad x \in P$ or $y \in P$ or $z \in P$.

But the ideal $Q=\left\{30 k: k \in \mathbb{Z}^{-}\right\}$is not a prime ideal of $\mathbb{Z}^{-}$, since $(-2)(-3)(-5)=-30 \in Q$ but $(-2) \notin Q,(-3) \notin Q$ and $(-5) \notin Q$.

Theorem 2.11. If $I$ is an ideal of a ternary semigroup $S$ and $P$ is a prime ideal of $S$, then $I \cap P$ is a prime ideal of $I$, considering $I$ as a ternary semigroup.

Proof. Clearly, $I \cap P$ is an ideal of $I$. Let $a, b, c \in I$ and $a S b S c \subseteq I \cap P$, $a S S b S S c \subseteq I \cap P, a S S b S c S \subseteq I \cap P$ and $S a S b S S c \subseteq I \cap P$. Then $a S b S c \subseteq P, a S S b S S c \subseteq P, a S S b S c S \subseteq P$ and $S a S b S S c \subseteq P$, since $I \cap P \subseteq P$. Since $P$ is a prime ideal of $S$, we have $a \in P$ or $b \in P$ or $c \in P$. Thus $a \in I \cap P$ or $b \in I \cap P$ or $c \in I \cap P$. Consequently, by Theorem 2.8, $I \cap P$ is a prime ideal of $I$.

Note 2.12. Let $\left\{P_{i}\right\}$ be a collection of prime ideals of a ternary semigroup $S$. Then $\bigcup P_{i}$ and $\cap P_{i}$ are ideals of $S$ but these are not prime ideals of $S$, in general.

However; in particular, we have the following result:

Proposition 2.13. Let $\left\{P_{i}\right\}$ be a collection of prime ideals of a ternary semigroup $S$ such that $\left\{P_{i}\right\}$ forms a chain. Then $\bigcup P_{i}$ and $\bigcap P_{i}$ are both prime ideals of $S$.

Proof. Clearly, $\bigcap P_{i}$ is an ideal of $S$. Let $A B C \subseteq \bigcap P_{i}$ for any three ideals $A, B, C$ of $S$. If either $A \subseteq P_{i}$, for all $i$ or $B \subseteq P_{i}$, for all $i$ or $C \subseteq P_{i}$, for all $i$, then either $A \subseteq \bigcap P_{i}$ or $B \subseteq \bigcap P_{i}$ or $C \subseteq \bigcap P_{i}$. If possible, let $A, B, C \nsubseteq \bigcap P_{i}$. Then there exist $i, j$ and $k$ such that $A \nsubseteq \bigcap P_{i}, B \nsubseteq \bigcap P_{j}$ and $C \nsubseteq \cap P_{k}$. Since $\left\{P_{i}\right\}$ is a chain, let $P_{i} \subseteq P_{j} \subseteq P_{k}$. This implies that $B, C \nsubseteq P_{i}$. Since $A B C \subseteq P_{i}$ and $P_{i}$ is prime, we must have either $A \subseteq P_{i}$ or $B \subseteq P_{i}$ or $C \subseteq P_{i}$, a contradiction. Therefore, either $A \subseteq \bigcap P_{i}$ or $B \subseteq \bigcap P_{i}$ or $C \subseteq \bigcap P_{i}$. Consequently, $\bigcap P_{i}$ is a prime ideal of $S$.

Similarly, we can prove that $\bigcup P_{i}$ is a prime ideal of $S$.

Definition 2.14. A proper ideal $Q$ of a ternary semigroup $S$ is called a semiprime ideal of $S$ if $I^{3} \subseteq Q$ implies $I \subseteq Q$ for any ideal $I$ of $S$. 
Note 2.15. Every prime ideal of a ternary semigroup $S$ is also a semiprime ideal of $S$.

As in the case of prime ideals of a commutative ternary semigroup, we have the following result for semiprime ideals of a commutative ternary semigroup:

Theorem 2.16. A proper ideal $Q$ of a commutative ternary semigroup $S$ is semiprime if and only if $x^{3} \in Q$ implies that $x \in Q$ for any element $x$ of $S$.

Example 2.17. In the commutative ternary semigroup $\mathbb{Z}^{-}$of all negative integers, the ideal $Q=\left\{6 k: k \in \mathbb{Z}^{-}\right\}$is a semiprime ideal.

$$
\begin{array}{ll}
\text { For } & x^{3} \in Q\left(x \in \mathbb{Z}^{-}\right) \\
\Longleftrightarrow & x^{3} \text { is divisible by } 6 \\
\Longleftrightarrow & x \text { is divisible by } 6 \\
\Longleftrightarrow & x=6 k_{1} \text { for } k_{1} \in \mathbb{Z}^{-} \\
\Longleftrightarrow & x \in Q .
\end{array}
$$

Definition 2.18. A proper ideal $I$ of a ternary semigroup $S$ is said to be weakly irreducible if for ideals $H$ and $K$ of $S, H \cap K=I$ implies that $I=H$ or $I=K$.

We simply use the term irreducible to mean weakly irreducible.

Definition 2.19. A proper ideal $I$ of a ternary semigroup $S$ is said to be strongly irreducible if for ideals $H$ and $K$ of $S, H \cap K \subseteq I$ implies that $H \subseteq I$ or $K \subseteq I$.

Note 2.20. It is to be noted here that a strongly irreducible ideal of a ternary semigroup $S$ is an irreducible ideal of $S$.

Definition 2.21. A non-empty subset $A$ of a ternary semigroup $S$ is called an $i$-system if $a, b \in A$ implies that $\langle a\rangle \cap\langle b\rangle \cap A \neq \emptyset$.

Theorem 2.22. The following conditions in a ternary semigroup $S$ are equivalent:

(i) I is a strongly irreducible ideal of $S$;

(ii) If for $a, b \in S ;\langle a\rangle \cap<b>\subseteq I$ then $a \in I$ or $b \in I$; 
(iii) The complement of I i.e. $I^{c}$ is an i-system.

Proof. $(i) \Longrightarrow(i i)$.

This is an immediate consequence of the Definition 2.19.

(ii) $\Longrightarrow($ iii).

If possible let $a, b \in I^{c}$ and $\langle a\rangle \cap\langle b\rangle \cap I^{c}=\emptyset$.

Then $\langle a\rangle \cap\langle b\rangle \cap I^{c}=\emptyset$ implies that $\langle a\rangle \cap\langle b\rangle \subseteq I$ and hence by using (ii), we have $a \in I$ or $b \in I$, which is a contradiction.

Consequently, $\langle a\rangle \cap\langle b\rangle \cap I^{c} \neq \emptyset$ and hence $I^{c}$ is an $i$-system.

(iii) $\Longrightarrow($ i $)$.

Let $H$ and $K$ be two ideals of $S$ such that $H \nsubseteq I$ and $K \nsubseteq I$.

Then there exist elements $a \in H-I$ and $b \in K-I$. Now from (iii), it follows that $\langle a\rangle \cap\langle b\rangle \cap I^{c} \neq \emptyset$ i.e. there exists an element $c \in(\langle a\rangle \cap\langle b\rangle)-I$. This implies that $c \in H \cap K$ and $c \notin I$. Hence $H \cap K \nsubseteq I$. This shows that $I$ is strongly irreducible.

Theorem 2.23. A proper ideal $P$ of a ternary semigroup $S$ is prime if and only if it is semiprime and strongly irreducible.

Proof. Suppose $P$ is a prime ideal of $S$. Then $P$ is a semiprime ideal of $S$. Again, let $H$ and $K$ be two ideals of $S$ such that $H \cap K \subseteq P$. Now $S H K \subseteq S S K \subseteq K$ and $S H K \subseteq S H S \subseteq H$. This implies that $S H K \subseteq H \cap K \subseteq P$. Since $P$ is a prime ideal of $S$, it follows that $H \subseteq P$ or $K \subseteq P$ and hence $P$ is strongly irreducible.

Conversely, suppose that a proper ideal $P$ of $S$ is both semiprime and strongly irreducible. Let $A B C \subseteq P$ for any three ideals $A, B, C$ of $S$. Now $[(A \cap B) \cap C][(A \cap B) \cap C][(A \cap B) \cap C] \subseteq A B C \subseteq P$. Since $P$ is a semiprime ideal of $S$, it follows that $[(A \cap B) \cap C] \subseteq P$. Again, since $P$ is a strongly irreducible ideal, we have $A \cap B \subseteq P$ or $C \subseteq P$ which again implies that $A \subseteq P$ or $B \subseteq P$ or $C \subseteq P$. Consequently, $P$ is a prime ideal of $S$.

Definition 2.24. A subset $Q$ of a ternary semigroup $S$ is called a quasiideal of $S$ if $Q S S \cap S Q S \cap S S Q \subseteq Q$ and $Q S S \cap S S Q S S \cap S S Q \subseteq Q$.

Lemma 2.25. Every left, right, lateral ideal of a ternary semigroup $S$ is a quasi-ideal of $S$.

Remark 2.26. The converse of Lemma 2.25 is not true, in general i.e. a quasi-ideal may not be a left, a right or a lateral ideal of $S$. This follows from the following example. 
Example 2.27. Let $S=M_{2}\left(\mathbb{Z}_{0}^{-}\right)$be the ternary semigroup of the set of all $2 \times 2$ square matrices over $\mathbb{Z}_{0}^{-}$, the set of all non-positive integers. Let $Q=\left\{\left(\begin{array}{ll}a & 0 \\ 0 & 0\end{array}\right): a \in \mathbb{Z}_{0}^{-}\right\}$. Then we can easily verify that $Q$ is a quasi-ideal of $S$ but $Q$ is not a right ideal, a lateral ideal or a left ideal of $S$.

Proposition 2.28. The intersection of arbitrary collection of quasiideals of a ternary semigroup $S$ is a quasi-ideal of $S$.

Theorem 2.29. A subset $Q$ of a ternary semigroup $S$ is a quasi-ideal of $S$ if and only if $Q$ is the intersection of a right ideal, a lateral ideal and a left ideal of $S$.

Corollary 2.30. Every quasi-ideal of a ternary semigroup $S$ is a ternary subsemigroup of $S$.

Definition 2.31. A subsemigroup $B$ of a ternary semigroup $S$ is called a bi-ideal of $S$ if $B S B S B \subseteq B$.

Lemma 2.32. Every quasi-ideal of a ternary semigroup $S$ is a bi-ideal of $S$.

Note 2.33. The converse of Lemma 2.32 does not hold, in general i.e. a bi-ideal of a ternary semigroup $S$ may not be a quasi-ideal of $S$.

Remark 2.34. Since every left, right and lateral ideal of $S$ is a quasiideal of $S$, it follows that every left, right and lateral ideal of $S$ is a bi-ideal of $S$ but the converse is not true, in general.

In general, if $B$ is a bi-ideal of a ternary semigroup $S$ and $C$ is a bi-ideal of $B$ then $C$ is not a bi-ideal of $S$. But in particular, we have the following result:

Theorem 2.35. Let $B$ be a bi-ideal of a ternary semigroup $S$ and $C$ a bi-ideal of $B$ such that $C^{3}=C$. Then $C$ is a bi-ideal of $S$.

Proof. Since $B$ is a bi-ideal of $S, B S B S B \subseteq B$ and since $C$ is a biideal of $B, C B C B C \subseteq C$. Therefore, $C S C S C=(C C C) S C S(C C C)=$ $C C(C S C S C) C C \subseteq C C(B S B S B) C C \subseteq C C B C C=C C B C(C C C) \subseteq$ $C(C B C B C) C \subseteq C C C=C$. Thus $C$ is a bi-ideal of $S$. 
Definition 2.36. A ternary semigroup $S$ is called a ternary group if for all $a, b, c \in S$, the equations $a b x=c, a y b=c$ and $z a b=c$ have solutions in $S$.

Remark 2.37. In a ternary group $S$, for all $a, b, c \in S$, the equations $a b x=c, a y b=c$ and $z a b=c$ have unique solutions in $S$.

Theorem 2.38. A ternary semigroup $S$ has no proper bi-ideal if and only if $S$ is a ternary group.

Proof. Let $S$ be a ternary group. Let $B$ be a bi-ideal of $S$. Suppose that $c \in S$ and $a, b \in B$. Since $S$ is a ternary group, we have $x a b=c$ has a solution in $S$. This implies that $c=y a b$ for some $y \in S$. Consequently, $S=S B B$. Similarly, $S=B B S$ and $S=B S B$. Now $S=B B S=$ $B B S B B=B B B S B B B \subseteq B B(B S B S B) \subseteq B B B \subseteq B$. Consequently, $B=S$ and hence $S$ has no proper bi-ideal.

Conversely, suppose that $S$ contains no proper bi-ideals. Now for any $a, b, c \in S ; a b S, a S b$ and $S a b$ are all bi-ideals of $S$ and hence $a b S=a S b=$ $S a b=S$. This implies that the equations $a b x=c, a y b=c$ and $z a b=c$ have solutions in $S$. Consequently, $S$ is a ternary group.

Definition 2.39. An element $a$ in a ternary semigroup $S$ is called regular if there exists an element $x$ in $S$ such that $a x a=a$.

A ternary semigroup $S$ is called regular if all of its elements are regular.

Theorem 2.40. The following conditions in a ternary semigroup $S$ are equivalent:

(i) $S$ is regular;

(ii) For any right ideal $R$, lateral ideal $M$ and left ideal $L$ of $S, R M L=$ $R \cap M \cap L$;

(iii) For $a, b, c \in S$; $\left\langle a>_{r}<b>_{m}<c>_{l}=<a>_{r} \cap<b>_{m} \cap<c>_{l}\right.$;

(iv) For $a \in S$; $\left\langle a>_{r}<a>_{m}<a>_{l}=<a>_{r} \cap<a>_{m} \cap<a>_{l}\right.$.

Theorem 2.41. If for every quasi-ideal $Q$ of $S, Q^{3}=Q$ then $S$ is a regular ternary semigroup.

Proof. If $R$ is a right ideal, $M$ a lateral ideal and $L$ a left ideal of $S$, then by Theorem 2.29, it follows that $R \cap M \cap L$ is a quasi-ideal of $S$. Now by hypothesis,

$R \cap M \cap L=(R \cap M \cap L)^{3}=(R \cap M \cap L)(R \cap M \cap L)(R \cap M \cap L) \subseteq R M L$. 
Again, clearly we have $R M L \subseteq R \cap M \cap L$. Consequently, $R \cap M \cap L=$ $R M L$ and hence by Theorem 2.40, $S$ is a regular ternary semigroup.

The following theorem gives a characterization of a regular ternary semigroup $S$ in terms of bi-ideal and quasi-ideal of $S$.

Theorem 2.42. The following conditions in a ternary semigroup $S$ are equivalent:

(i) $S$ is regular;

(ii) For every bi-ideal $B$ of $S, B S B S B=B$;

(iii) For every quasi-ideal $Q$ of $S, Q S Q S Q=Q$.

Proof. $(i) \Longrightarrow(i i)$

Suppose $S$ is regular. Let $B$ be a bi-ideal of $S$. Let $b \in B$. Then there exists $x \in S$ such that $a=a x a$. This implies that $a=\operatorname{axaxa} \in B S B S B$. So we find that $B \subseteq B S B S B$. Again, since $B$ is a bi-ideal of $S, B S B S B \subseteq B$. Consequently, we have $B S B S B=B$.

Clearly, $($ ii $) \Longrightarrow($ iii $)$, by using Lemma 2.32 .

(iii) $\Longrightarrow($ i $)$

Suppose (iii) holds. Let $R$ be a right ideal, $M$ a lateral ideal and $L$ a left ideal of $S$. Then $Q=R \cap M \cap L$ is a quasi-ideal of $S$, by Theorem 2.29. By hypothesis, $Q S Q S Q=Q$. Now $R \cap M \cap L=Q=Q S Q S Q \subseteq R S M S L \subseteq$ $R M L$. Again, clearly $R M L \subseteq R \cap M \cap L$. So $R \cap M \cap L=R M L$ and hence by Theorem $2.40, S$ is a regular ternary semigroup.

Theorem 2.43. A ternary subsemigroup $B$ of a regular ternary semigroup $S$ is a bi-ideal of $S$ if and only if $B=B S B$.

Proof. If $B=B S B$, then it is easy to see that $B$ is a bi-ideal of $S$.

Conversely, suppose that $B$ is a bi-ideal of a regular ternary semigroup $S$. Let $b \in B$. Then there exists $x \in S$ such that $b=b x b$. This implies that $b \in B S B$ and hence $B \subseteq B S B$. Again, $B S B \subseteq B S B S B \subseteq B$. Thus we find that $B=B S B$.

Theorem 2.44. A subsemigroup $B$ of a regular ternary semigroup $S$ is a bi-ideal of $S$ if and only if $B$ is a quasi-ideal of $S$.

Proof. Let $S$ be a regular ternary semigroup. If $B$ is a quasi-ideal of $S$, then from Lemma 2.32, it follows that $B$ is a bi-ideal of $S$. 
Conversely, let $B$ be a bi-ideal of $S$. From Theorem 2.40, we find that if $S$ is a regular ternary semigroup, then $R \cap M \cap L=R M L$ for any right ideal $R$, any lateral ideal $M$ and any left ideal $L$. Now

$$
\begin{aligned}
& B S S \cap(S B S \cup S S B S S) \cap S S B=B S S(S B S \cup S S B S S) S S B \\
& =B(S S S) B(S S S) B \cup B(S S S) S B(S S S) S B \subseteq B S B S B \cup B S S B S S B \\
& \subseteq B \cup B S B \quad \quad \text { (since B is a bi-ideal) } \\
& =B \cup B \quad \text { (by Theorem 2.42) } \\
& =B .
\end{aligned}
$$

Consequently, $B$ is a quasi-ideal of $S$.

From Theorem 2.29 and Theorem 2.44, we have the following result:

Corollary 2.45. A subsemigroup $B$ of a regular ternary semigroup $S$ is a bi-ideal of $S$ if and only if $B$ is the intersection of a right ideal, a lateral ideal and a left ideal of $S$.

Definition 2.46. A subsemigroup $B_{w}$ of a ternary semigroup $S$ is called a weak bi-ideal of $S$ if $b S b S b \subseteq B_{w}$, for all $b \in B_{w}$.

Proposition 2.47. Every bi-ideal of a ternary semigroup $S$ is a weak bi-ideal of $S$.

Remark 2.48. Since every left ideal, right ideal and lateral ideal of $S$ is a bi-ideal of $S$, it follows that every left ideal, right ideal and lateral ideal of $S$ is a weak bi-ideal of $S$.

The converse of the above result is not true. This follows from the following example:

Example 2.49. Let $S=\mathbb{Z}^{-} \times \mathbb{Z}^{-}=\left\{(a, b): a, b \in \mathbb{Z}^{-}\right.$, set of all negative integers\}.

Then $S$ is a ternary semigroup w.r.t. the ternary multiplication defined as follows: $(a, b)(c, d)(e, f)=(a, f)$.

Let $B_{w}=\{(b, b):(b, b) \in S\}$. Then $B_{w}$ is a weak bi-ideal of $S$, since $(b, b)(u, v)(b, b)(x, y)(b, b)=(b, b) \in B_{w}$, for all $(u, v),(x, y) \in S$. It can be easily verified that $B_{w}$ is neither a bi-ideal nor a left ideal, a right ideal and a lateral ideal of $S$.

Proposition 2.50. The intersection of arbitrary collection of weak biideals of a ternary semigroup $S$ is a weak bi-ideal of $S$. 
Theorem 2.51. A ternary semigroup $S$ is regular if and only if $B_{w}=$ $\bigcup_{b \in B_{w}} b S b S b$, where $B_{w}$ is a weak bi-ideal of $S$.

Proof. Let $S$ be a regular ternary semigroup and $B_{w}$ be a weak bi-ideal of $S$. Clearly, $\bigcup_{b \in B_{w}} b S b S b \subseteq B_{w}$. Now let $b \in B_{w}$. Since $S$ is regular, we have $b=b x b$ for some $x \in S$. So $b=b x b=b x b x b \in \bigcup_{b \in B_{w}} b S b S b$. Thus $B_{w} \subseteq \bigcup_{b \in B_{w}} b S b S b$ and hence $B_{w}=\bigcup_{b \in B_{w}} b S b S b$.

Conversely, suppose that $B_{w}=\bigcup_{b \in B_{w}} b S b S b$ for any weak bi-ideal $B_{w}$ of $S$. Let $R$ be a right ideal, $M$ be a lateral ideal and $L$ be a left ideal of $S$. Then $R, M, L$ are weak bi-ideals of $S$. Consequently, we find that $R \cap M \cap L$ is a weak bi-ideal of $S$, by Proposition 2.50. Clearly, $R M L \subseteq R \cap M \cap L$. Let $a \in R \cap M \cap L$. Then $a=b s b t b$ for some $b \in R \cap M \cap L$ and $s, t \in S$. This implies that $a=b s b t b \in R M L$. So $R \cap M \cap L \subseteq R M L$ and hence $R M L=R \cap M \cap L$. Consequently, $S$ is a regular ternary semigroup, by Theorem 2.40 .

Diagrammatic representation of different ideals of ternary semigroups

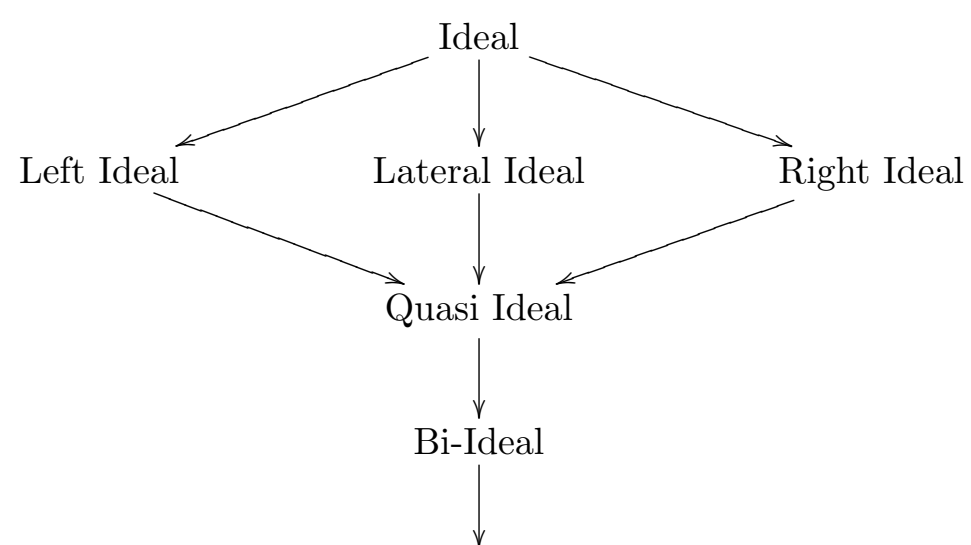

Weak Bi-Ideal

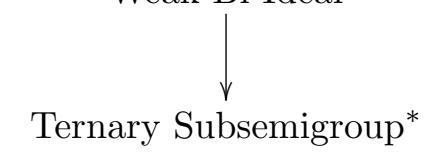

*The reverse implication does not always hold.

Prime Ideal $\Longleftrightarrow$ Semiprime Ideal + Strongly Irreducible Ideal. 


\section{REFERENCES}

1. Clifford, A.H.; Preston, G.B. - The Algebraic Theory of Semigroups, Vol. I. Mathematical Surveys, No. 7, American Mathematical Society, Providence, R.I., 1961.

2. Clifford, A.H.; Preston, G.B. - The Algebraic Theory of Semigroups, Vol. II. Mathematical Surveys, No. 7, American Mathematical Society, Providence, R.I., 1967.

3. Dixit, V.N.; Dewan, S. - A note on quasi and bi-ideals in ternary semigroups, Internat. J. Math. Math. Sci., 18 (1995), 501-508.

4. DutTA, T.K.; KAR, S.; MAITY, B.K. - On ideals in regular ternary semigroups, Discuss. Math. Gen. Algebra Appl., 28 (2008), 147-159.

5. HowIE, J.M. - Fundamentals of semigroup theory, London Mathematical Society Monographs, New Series, 12, Oxford Science Publications, The Clarendon Press, Oxford University Press, New York, 1995.

6. KAR, S.; MAITY, B.K. - Congruences on ternary semigroups, Journal of the Chungcheong Mathematics Society, 20 (2007), 191-201.

7. Łoś, J. - On the extending of models. I, Fund. Math., 42 (1955), 38-54.

8. Santiago, M.L. - Some contribution to the study of ternary semigroups and semiheaps, Ph.D. Thesis, University of Madras, India, 1983.

9. Sioson, F.M. - Ideal theory in ternary semigroups, Math. Japon., 10 (1965), 63-84.

Received: 16.III.2009

Department of Mathematics, Jadavpur University, Kolkata, 700032, West Bengal, INDIA

karsukhendu@yahoo.co.in bkmaity2002@yahoo.co.in 